\title{
From theory to practice: a conceptual framework to facilitate implementation of evidence in stroke rehabilitation for local context in Saudi Arabia
}

This article was published in the following Dove Press journal:

Journal of Multidisciplinary Healthcare

\author{
Salem F Alatawi \\ Department of Physical Therapy, Faculty \\ of Applied Medical Sciences, University of \\ Tabuk, Tabuk City, Kingdom of Saudi \\ Arabia
}

Introduction: Implementing evidence into practice is a global priority with implications for patients, researchers, practitioners and policy-makers (stakeholders). The national clinical guideline $(\mathrm{NCG})$ for stroke is one of the most important sources of robust evidence. However, implementation within real practice is often slow to respond. The knowledge to action (KTA) framework is one of several frameworks to facilitate implementation and enable evidence to be put into operation. This study aims to develop a conceptual framework to understand the process of implementation of an NCG for stroke in the local context of physiotherapy practice in the Kingdom of Saudi Arabia.

Methods: A qualitative-quantitative mixed methods study was conducted. The first method (panel meetings, 10 expert physiotherapists) was to chart the main concepts/domains of physiotherapy practice in the UK NCG for stroke. Drawing on panel meeting findings, 75 recommendations were released as an online questionnaire to 35 Saudi stroke specialist physiotherapists. This was followed by a focus group (second method) to capture the process of implementation of NCG for stroke in the Kingdom of Saudi Arabia.

Results: Sixty-one recommendations were accepted to be applied in real practice, whereas only 14 recommendations were rejected. The paper presents the first empirically derived framework that establishes thecontributionof physiotherapy to stroke rehabilitation. This framework characterizes the real-world nature of the implementation of NCGs for stroke within physiotherapy practice. This framework reaffirms the importance of supportive organizational culture, the specific need of end users, patient factors, and finally transferability of the evidence.

Discussion: This conceptual framework appears to provide a reasonable approach for the development of implementation strategies for physiotherapist practice in stroke rehabilitation. Furthermore, it might be the first that reflects the real value of the KTA framework and tests it empirically.

Conclusion: The finding of this study provides a useful comprehensive framework to implement existing NCGs for stroke.

Keywords: physiotherapists/physical therapists practice, stroke rehabilitation, knowledge translation, national clinical guidelines, implementation

\section{Introduction}

Implementation of evidence-based practice (EBP) in the healthcare context is a tasking activity that is characterized by implementation processes, such as individual and/or organizational practice. The current variation in practice indicates the need to improve or change practice using theories and frameworks, as they help
Department of Physical Therapy, Faculty of Applied Medical Sciences, University of Tabuk, P.O. Box 74I, 7I49I, Tabuk City,

Kingdom of Saudi Arabia

Tel +966 I4 4563434

Email sfalatawi@ut.edu.sa
Journal of Multidisciplinary Healthcare 2019:12 515-525 
to understand and execute the implementation process. ${ }^{1}$ A number of theories found in the literature are adopted for specific implementation interventions at different levels; namely, individual, group, and organisation levels. The complexity of the implementation of evidence in health care at different levels indicates the need to use more than one theory in order to record successful and positive outcomes from the process. ${ }^{1}$

In terms of physiotherapy practice, findings ${ }^{2-14}$ revealed that most physiotherapists had positive attitudes towards the implementation process. However, it is important to note that positive attitudes toward implementation were linked to other factors such as skills, difficulty using databases, experience, lack of time, training, research skills, understanding of the terminology reported in papers, high workload, funding, and employment sectors. Other authors showed that patient factors, lack of confidence in the validity of research findings, and in the transferability of research findings to an individual's working environment were most common barriers to the implementation process in physiotherapy practice. ${ }^{15-20}$ Whereas studies reported mixed effects in the implementation of evidence into practice, a study conducted by Scott et $\mathrm{al}^{21}$ revealed that most implementation strategies used educational meetings in allied health. In addition, some implemented multiple interventions.
Several conceptual frameworks can be used to facilitate the implementation process and record increased instances of practice change and spread of evidence. ${ }^{22-24}$ Stetler's model ${ }^{25}$ is one of the first models applied to the implementation of evidence-based research, despite its failure to examine wider organizational issues and the organization's impact on the implementation process. This was followed by the release of other models/frameworks for the implementation process. Examples of such frameworks include the Ottawa Model of Research Use,26 the Promoting Action on Research Implementation in Health Services (PARIHS), ${ }^{27,28}$ the Consolidated Framework for Implementation Research (CFIR), ${ }^{29}$ and the knowledge to action framework (KTA). ${ }^{30}$ However, there is little specificity that is currently available to provide guidance for the actual facilitation process, but the body is expected to grow with time.

Evidence from existing studies reports the need to understand the diversity of models and frameworks so that they are used appropriately for the target group, situation, and context. Of particular interest to this concept analysis is the KTA framework, which has two components: knowledge creation, and action cycle (Figure 1).

The center of the KTA framework is the knowledge funnel, which represents knowledge creation. However, knowledge creation, or production of knowledge, consists

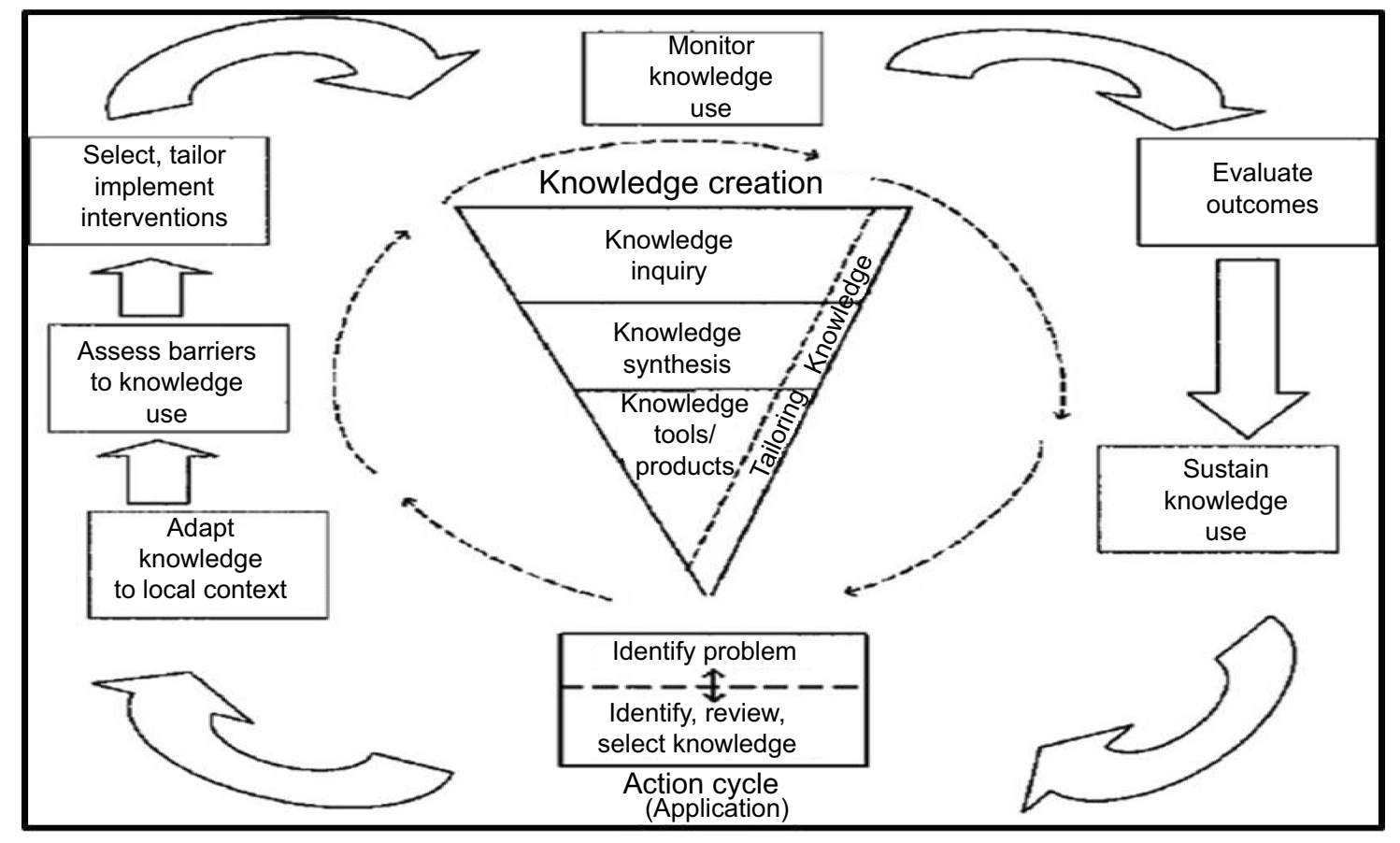

Figure I Knowledge to action (KTA) framework. 
of three phases: knowledge inquiry, knowledge synthesis, and knowledge tools and/or product creation. As knowledge moves through the funnel, it is refined and, ideally, becomes more useful to end users of the knowledge. At the end of this funnel, a small number of tools or products present evidence in concise and user-friendly formats tailored to meet enduser informational needs. These include national clinical guidelines (NCGs). The second part of the KTA cycle is the action cycle. The action cycle includes a range of activities needed for knowledge implementation. The action cycle is iterative and includes the deliberate application of knowledge to cause change in behaviors and/or attitudes.

In stroke, the main focus on implementation is the production of evidence-based guideline for practitioners to use. Many countries now have an NCG for stroke. The primary goals of clinical guidelines include decreasing the variability and increasing transparency in clinical practice, and legitimization of the profession. ${ }^{31,32}$ However, in practice such guidelines are rarely straightforward or predictable. Studies ${ }^{31-35}$ reported that clinical guidelines and recommendations did not yield standardized care approaches. In this case, the focus was pegged on the production of evidencebased guidelines to be used by practitioners. Despite the fact that most countries have an NCG for stroke, many practitioners are not aware of strategies to enhance their usage of the guidelines in practice.
The purpose of this study was to develop a conceptual framework that could be used to implement stroke rehabilitation in the Saudi Arabian context. The completed framework aimed to add value to the effectiveness and successful process in the implementing of EPB. The KTA framework was used as a guiding model to help elucidate key elements of the implementation process.

\section{Methods}

Qualitative-quantitative mixed methods (Figure 2) study were conducted, including a survey and focus group meeting.

A panel group meeting was used to determine the treatment areas of physiotherapy contributing to stroke rehabilitation, and to filter the UK NCG for stroke - 5th edition, 2016. In relation to physiotherapy contribution in stroke rehabilitation, followed by creating a questionnaire. A focus group meeting technique was conducted to capture the process of tailoring and implantation of recommendations of the NCG for stroke rehabilitation. This allows participants to discuss issues face to face, and the structured process is believed to facilitate contributions from all group members and to limit dominance by eminent or eloquent individuals. Permission to conduct the research was obtained from the research ethics committee, University of Tabuk, Kingdom of Saudi Arabia. All the participants who agreed to participate were required to sign an informed consent form. An informed consent

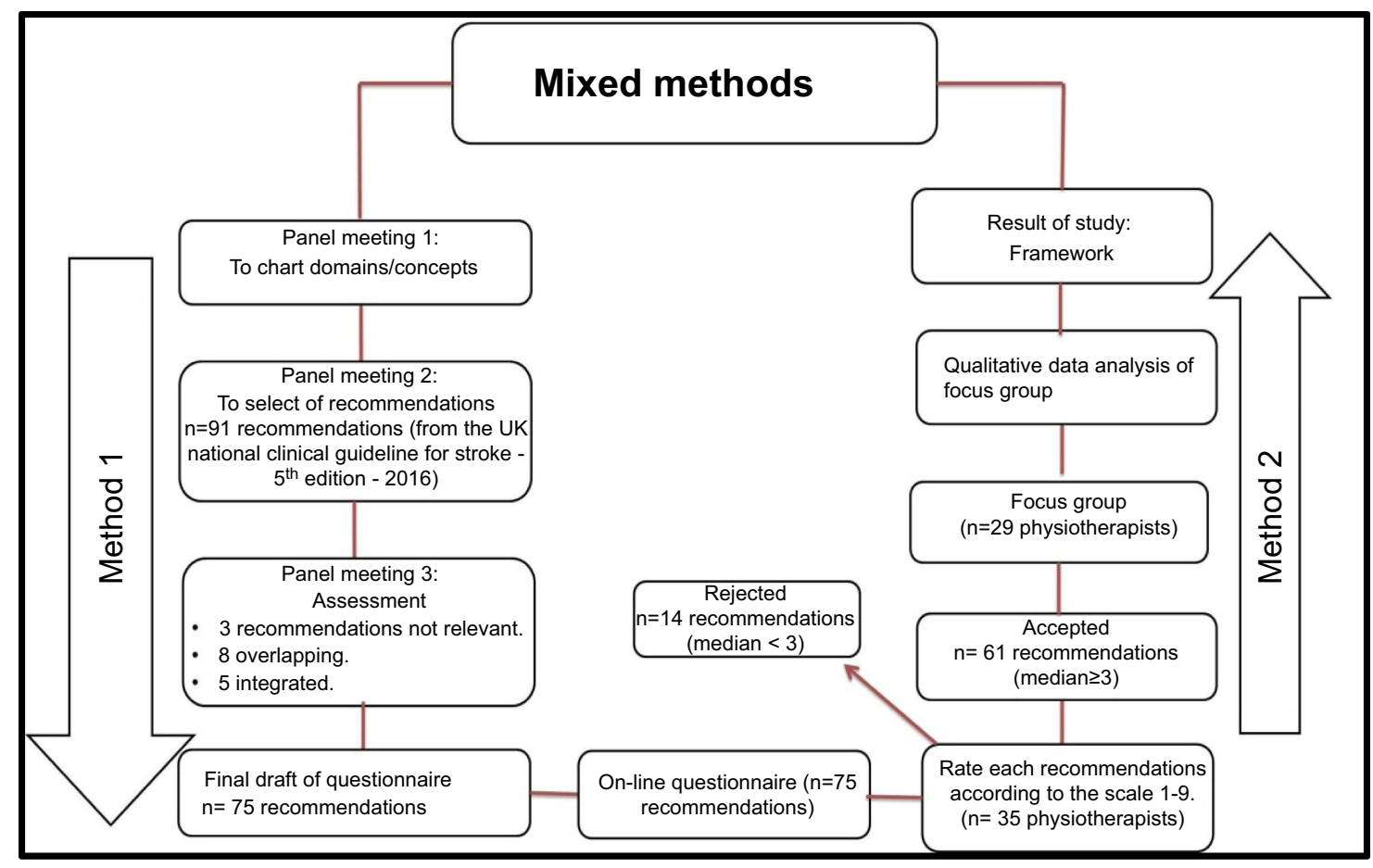

Figure 2 A plan chart of the study method. 
package included an information sheet detailing the aim of the research and what taking part involved.

\section{Expert panel meeting}

The first phase involved engaging 10 physiotherapists in stroke rehabilitation, by introducing the project via a rolling presentation program. The participants reflected the full range of people to which the guideline will apply; for example, individuals with expertise in stroke rehabilitation, academic people, and physiotherapists.

Data were collected to chart domains of practice in relation to stroke intervention in the participating physiotherapists in stroke rehabilitation.

Drawing on the findings from the panel group meeting, four treatment areas (domains) were nominated; these included prevention, assessment, treatment, and delivery of services. They covered a total of 78 concepts around the contribution of physiotherapy to stroke rehabilitation.

\section{Expert panel selection of items}

After juxtaposition of the NCG recommendations (423 recommendations) in a horizontal column and all key concepts (78 items) in a vertical column, a tick was placed in the
Excel table for each recommendation in horizontal column was reversed to any concept in vertical column (Figure 3).

Eventually, 91 recommendations from NCG recommendations in the horizontal column were crossed with 78 key concepts in the vertical column.

\section{Assessment of items}

The experts were asked to check the first draft of the questionnaire. They were asked whether they think items of NCG of the desirable issue about the physiotherapy contribution in stroke rehabilitation were lacking. The experts were also asked to find items in NCGs which might overlap with each other.

Sixteen recommendations were excluded after assessment and discussion by the experts as follows: 3 items were thought not relevant, 8 items overlapped each other, and 5 were integrated with included items. Consequently, the final version of the questionnaire comprised 75 questions.

\section{Online questionnaire}

Seventy-five recommendations (items) from NCG recommendations were categorized into the main domains and related concepts of the findings of the panel, and put in a table. Each recommendation was rated 1-9 on a Likert

List of the UK national clinical guideline for stroke (5 $5^{\text {th }}$ edition-2016)

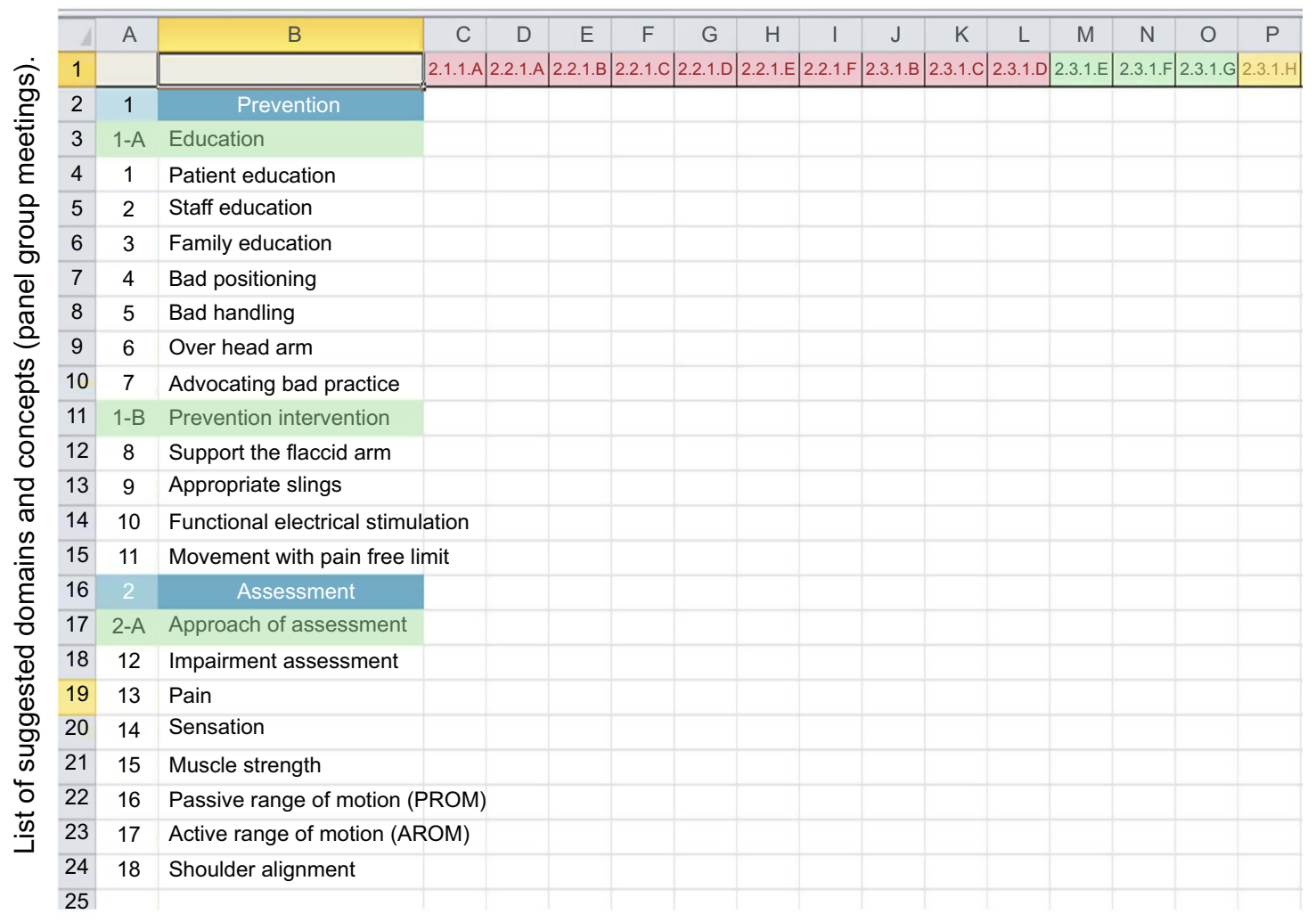

Figure 3 Excel sheet that shows cross between national clinical guideline for stroke (horizontal column) and key domains/concepts of panel meeting (vertical column). 


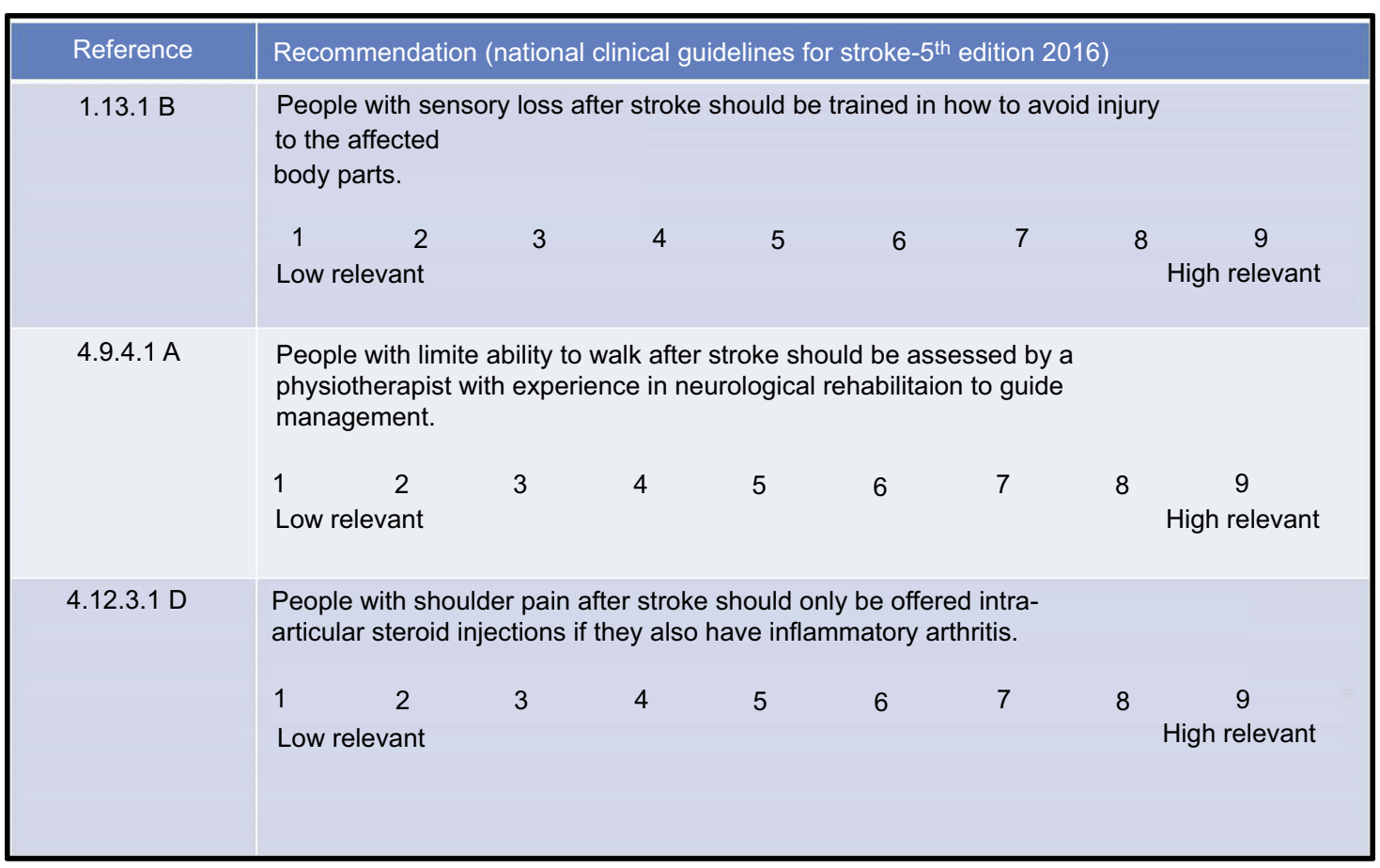

Figure 4 This part of the questionnaire asks participants to think about how relevant potential recommendations of national clinical guidelines may be in the management of stroke patients. For each item, participants have been asked to rate how strongly they agree or disagree with each item being included in the real practice.

scale where 1 represented not relevant to physiotherapist practice in stroke rehabilitation and 9 represented highly relevant (Figure 4 for example).

The frequency, median scores, and inter-quartile ranges were calculated for each recommendation. This allowed participants an opportunity, during the forthcoming focus group meeting, to see the spread of agreement and how their response related to this. The first section of this questionnaire captured personal information about the respondent.

\section{Focus group meeting}

The recommendations which the participants had already rated via the online questionnaire were discussed in turn, focusing primarily on those that were the source of most agreement (tight range) or most disagreement (wide range) between participants to be relevant to their clinical practice (Figure 5) to be included in physiotherapy practice. Some of these recommendations were subjected to detailed scrutiny and discussion between participants in a focus group where participants were invited to comment on their relevance and feasibility in their practice in stroke rehabilitation.

\section{Sample}

The study sample for the questionnaire involved all practicing physiotherapists who are currently providing stroke rehabilitation services in Tabuk, Kingdom of Saudi Arabia. Only physiotherapists who completed the questionnaire with an interest in implementing EBP in stroke rehabilitation participated in the focus group. Based on Polit and Beck, ${ }^{38}$ sample size in qualitative research was determined based on the information needs, and the guiding principle is data saturation.

\section{Data collection}

Data were gathered sequentially: an online survey was completed initially, followed by the focus group with stroke specialist physiotherapists. A list of addresses and emails of physiotherapists who routinely work with stroke patients was provided by the manager of the physiotherapy stroke rehabilitation service.

\section{Data analysis}

\section{For the online questionnaire}

Statistical Package for the Social Sciences (SPSS, Version 23, IBM Corporation, Armonk, NY, USA) was used to analyze the questionnaire data. Median scores and interquartile ranges were calculated for each recommendation. If the median score of a statement was $<3$, this was rejected as not relevant to physiotherapy practice in stroke rehabilitation. 


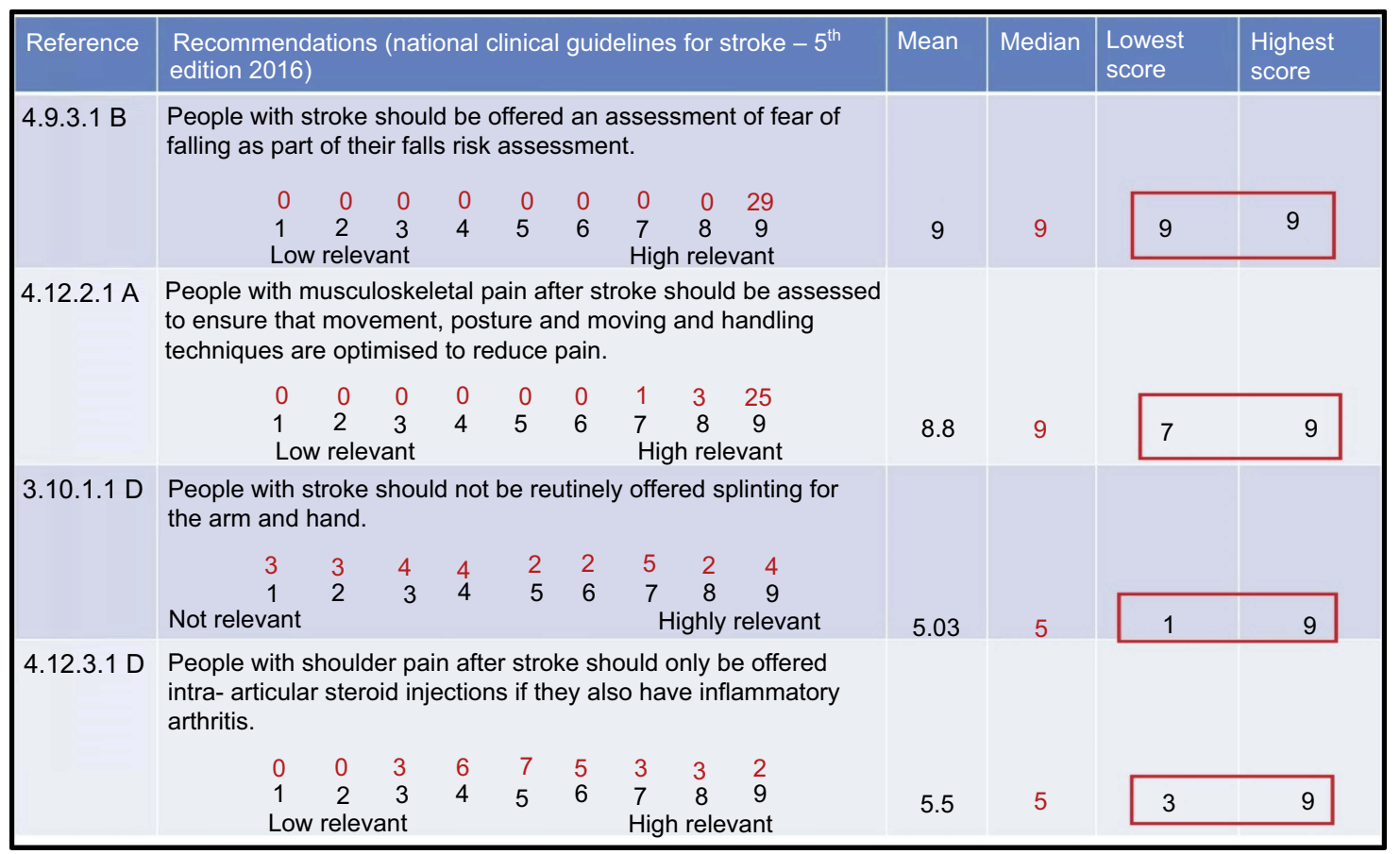

Figure 5 An example of the frequency of response to each recommendation was presented for the members of the focus group, focusing primarily on those that were the source of most agreement/disagreement. This allowed the challenges of implementing this recommendation in real practice to be understood.

Notes: Red box indicates to the spread of participants agreement about the recommendation; Red number indicates the rating of the individual participant on the recommendation of national clinical guideline for stroke.

\section{For the focus group}

Recordings of the focus group were transcribed and the transcripts were prepared for data analysis after conducting a manual quality check. Qualitative content analysis and thematic description yielded themes and issues, which were validated by two independent researchers using initial transcripts. Analysis of the transcripts was guided by the agreements and dissent evidenced in the focus group.

\section{Results}

\section{Findings of the survey}

Of the 35 questionnaires distributed, 29 were completed, representing an $83 \%$ response rate. The majority of participants were male $(58.6 \%)$ with the majority of the respondents $(44.8 \%)$ falling in the age category 20-29. Of the respondents in this study $51.7 \%$ had seen between 11 and 15 stroke patients per month. Demographic data are presented in Table 1. Sixty-one recommendations (items) form the UK NCG for stroke were thought to be relevant to physiotherapy practice because they met the criteria (that is, a median score $\geq 3$ ), whereas, 14 recommendations were rejected (Appendix 1).

\section{Findings of the focus group}

This step focused on the survey findings (method 1) as a precursor to discussing the local implementation context for physiotherapy within stroke services in the Kingdom of Saudi Arabia. A total sample of 29 physiotherapists working with stroke patients was included in this focus group. Four themes emerged from the qualitative analysis as relevant in implementing stroke rehabilitation evidence and improving clinical practice in the local context, Kingdom of Saudi Arabia. These factors are as follows: organizational factors, individual factors, patient factors, and lastly research factors.

\section{Organizational culture}

First, the organizational factors reveal the significance of the organizational structure in the implementation of EBP. Despite the fact that study participants established how the working environment supported the rehabilitation evidence, they reported organizational barriers that hindered the process, for instance, lack of time, financial constraints, pressure of work, inadequate numbers of staff with large numbers of patients, and failure to utilize available equipment and facilities. One of the physiotherapists reported that: 
Table I Demographic characteristics of the study participants

\begin{tabular}{|c|c|c|}
\hline QI: Gender & Frequency (n) & Valid percentage \\
\hline Male & 17 & $58.6 \%$ \\
\hline Female & 12 & $41.4 \%$ \\
\hline Total & 29 & $100 \%$ \\
\hline \multicolumn{3}{|l|}{ Q2: Age (years) } \\
\hline $20-29$ & 13 & $44.8 \%$ \\
\hline $30-39$ & 10 & $34.5 \%$ \\
\hline $40-49$ & 6 & $20.7 \%$ \\
\hline$<50$ & 0 & $0 \%$ \\
\hline Total & 29 & $100 \%$ \\
\hline \multicolumn{3}{|l|}{ Q3: Place of work } \\
\hline Government hospitals & 25 & $86.2 \%$ \\
\hline Private hospitals & 4 & $13.8 \%$ \\
\hline Total & 29 & $100 \%$ \\
\hline \multicolumn{3}{|c|}{ Q4: Experience in years } \\
\hline $1-5$ & 8 & $27.6 \%$ \\
\hline $6-10$ & 9 & $31.0 \%$ \\
\hline $11-15$ & 6 & $20.7 \%$ \\
\hline $16-20$ & 3 & $10.3 \%$ \\
\hline More than 20 & 3 & $10.3 \%$ \\
\hline Total & 29 & $100 \%$ \\
\hline \multicolumn{3}{|l|}{ Q6: Job title } \\
\hline Health assistant & 0 & $0 \%$ \\
\hline Technician & 3 & $10.3 \%$ \\
\hline Specialist & 18 & $62.1 \%$ \\
\hline Senior specialist & 5 & $17.2 \%$ \\
\hline Consultant & 3 & $10.3 \%$ \\
\hline Total & 29 & $100 \%$ \\
\hline \multicolumn{3}{|c|}{ Q7: Highest qualification } \\
\hline Diploma & 2 & $6.9 \%$ \\
\hline BSc & 19 & $65.5 \%$ \\
\hline DPT & 1 & $3.4 \%$ \\
\hline MSc & 5 & $17.2 \%$ \\
\hline $\mathrm{PhD}$ & 2 & $6.9 \%$ \\
\hline Other & 0 & $0 \%$ \\
\hline Total & 29 & $100 \%$ \\
\hline \multicolumn{3}{|c|}{ Q9: Number of patients/month } \\
\hline 5 & 1 & $3.4 \%$ \\
\hline $5-10$ & 9 & $31 \%$ \\
\hline $11-15$ & 15 & $51.7 \%$ \\
\hline 15 & 4 & $13.8 \%$ \\
\hline Total & 29 & $100 \%$ \\
\hline
\end{tabular}

It all depends on the work load at the time and the staffing issues. Ideally, it should be seen every day. However, you know, lack of time within working day could result in patients missing out on a rehabilitation, fail to complete treatment session and full use of available equipment and facilities.

The impact of resources upon physiotherapists' thinking was clear to include NCG for stroke in their practice about stroke rehabilitation. However, the study participants reported this dilemma disappeared with some recommendations as they did not require equipment for use in clinical practice.

I think that the other thing that sometimes limits staff is equipment. However, for some evidence, you just need your hand and your clinical knowledge.

In addition to drawing a clear line between the implementation of evidence in the local practice, physiotherapists outlined the impact of organizational factors, such as multidisciplinary teamwork, group discussion, and educational opportunities on their ways of thinking.

Our goals will be discussing as MDT every week, nurses, OTs, speech, physios, doctors, dietician, and pharmacies, so all will be discuss if there is anything for working incontinence.

\section{End users}

As one of the vital themes that resulted from the study, end users (therapist factors) provided information regarding how the practitioners reacted towards the clinical practice and in the process of implementing evidence in stroke rehabilitation. Study participants interviewed reported that skills varied between practitioners, which demonstrated the differences in individuals' knowledge base and perceptions. This includes relevant issues which can affect physiotherapists such as attitudes, behaviors, experience, understanding of terminology and views towards implementation of a particular intervention and the customs and practices which take place in a particular context. For example, study participants agreed that the hierarchies of evidence (such as RCTs) were of minimal relevance to physiotherapy. It was argued that evidence sought should be relevant to the clinical dilemma and the whole person.

The inclusion and exclusion criteria for an RCT can be so tight that actually they miss out all the patients that I'm actually trying it with.

The physiotherapists recognized clinical experience as an important component in enabling NCG for stroke as the practitioners have a high likelihood of using relevant 
information in the practice. One of the participants reported that:

If somebody who has been experienced can give the right idea at that time, but somebody who is not experienced, then they may have an idea how to deal with that.

Additionally, It was clear that physiotherapists who have experience, previous history or "previous exposure" to this intervention in the earlier time, are more likely to utilize relevant information and might use previously stored information in a specific way that encourages applying evidence with current situation. Obviously, participants confirmed that possessing stroke specialist expertise was key in building new knowledge needed in the practice.

It would be nice to have a specialist expertise in stroke rehabilitation. I've picked up from expertise I suppose, What is going on, can you come over to see this patient and struggling?

\section{Patient factors}

Participants reported that the inability of some of the stroke patients to participate in the treatment session compounded with the problem of understanding information as being their main concerns. Despite the fact some strategies could be utilized to manage the problems, for instance, talking to doctors and family members, there was the challenge of patients failing to describe the pain or use self-reported pain scales. In the interview, one of the participants stipulated that:

Depends on how patients present, a lot of that stroke patient will have complement problems. So finding out what works best for them and then see the way they will work with you.

On the other hand, it was revealed that physiotherapists experienced considerable difficulties when managing stroke due to the discrepancies where the home environment interventions differed with the clinical intervention when administering therapy. However, the participants reported the initiative undertaken to involve the client and the family in any stipulated rehabilitation program was a key component in stroke rehabilitation and might motivate the patient to engage easily in the treatment session.

If somebody had stroke, the role of family is important, because they are with the patient every day. So quite often we'll try and bring the carer in for treatment session, you know, how to transfer somebody safely, go through the exercise with them, because some stroke patients think why am I doing this, it's not relevant to me, but once they know the importance of it, much better results happened.

\section{Transferability of evidence}

Results from the study indicated that feasibility as one of the central issues in embedding evidence was pertinent in applying the evidence in the clinical practice with the challenge of applying certain interventions due to their technicalities.

When you look at the evidence you have to have it on for a high number of hours, and you have to keep putting it on, and actually when you take it off it doesn't show a carry.

Physiotherapists also reported the difficulty of applying some of the interventions in their current working environments. However, they noted that this particular intervention could be utilized in other different clinical locations privately. One of the participants reported that:

We can't offer it here, however if you know it works, you can see a private practitioner who can do it.

\section{Development of a conceptual framework to implemnt NCG for stroke}

Following a mixed methods study, a potential framework consisting of the four thematic areas (organizational culture, end users, patient factors and transferability of evidence) was developed in a bid to conceptualize the implementation process of stroke rehabilitation (Figure 6).

\section{Discussion}

The main purpose of this study was to develop a conceptual framework to implement EBP in stroke rehabilitation. This study used mixed methods to collect data from physiotherapists who worked closely with stroke patients. Interviews were focused on the challenges of getting evidence into practice and ascertaining what physiotherapy practice could offer in terms of implementing evidence into stroke rehabilitation.

Furthermore, this was the first paper that showed how the KTA framework can be used to facilitate the implementation of evidence in physiotherapy practice. For example, the KTA framework does not specifically prescribe what needs to be done at each phase. However, in this study, the idea of the knowledge funnel in the KTA framework was presented by NCG for stroke, whereas the 


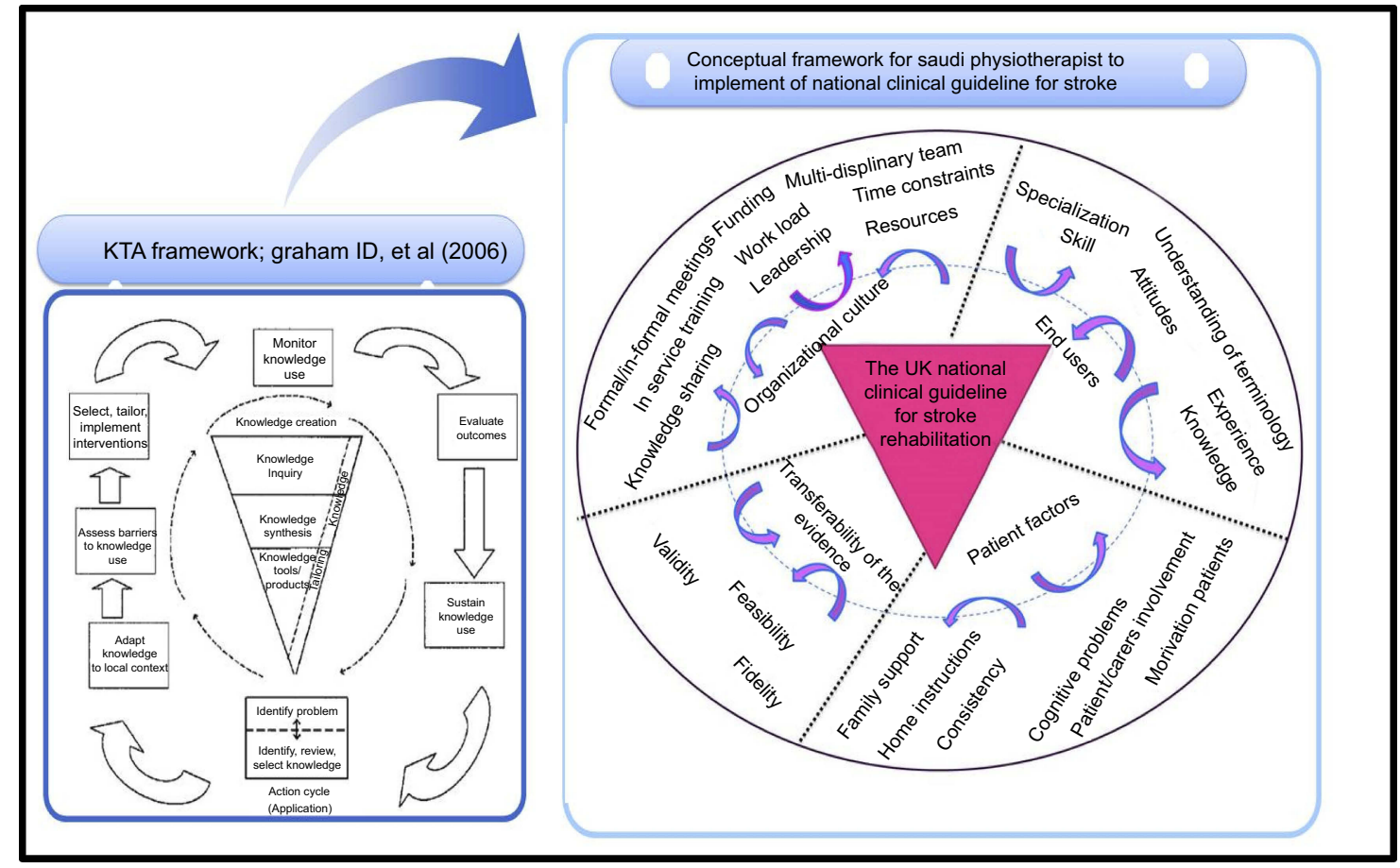

Figure 6 The conceptual framework for enhancing implemention of national clinical guidelines for stroke in real practice for stroke specialist physiotherapists in the Kingdom of Saudi Arabia. The idea of the first component of the KTA framework (knowledge creation) was used here by using the UK national clinical guideline for stroke. The second component of the KTA framework (action cycle) was clarified by using a focus group to identify and understand different challenges of implementation of national clinical guidelines for stroke in real physiotherapist practice.

action cycle was clarified by the conceptual framework, to discover how the implementation process may apply and facilitate at different levels in real clinical practice (Figure 6).

This framework involves findings that outline the implication of the implementation process in the work of healthcare professionals alongside the argument of the approach in physiotherapy.

\section{Organizational culture}

Evidence from this study showed that effective communication was considered important for improving the implementation process. These ideas match findings from previous studies, eg, a study proposed that a number of strategies existing in an organization, including education, discussion groups, and audit and feedback, could facilitate implementation. ${ }^{36}$

Further findings from the current study revealed that sharing information and carrying out consultations among colleagues or sharing specialist knowledge was recognized as crucial in the implementation process. This finding was consistent with findings from other studies conducted related to implementation and physiotherapy, which ascertained that research findings shared between physiotherapists in their current practice were used to guide decision-making and encouraged the implementation process. ${ }^{6,9}$ However, it was noted that a lack of physiotherapists with the necessary skills, knowledge, and experience could limit the practice of sharing information.

In general, personal and environmental factors played an essential role in the delivery of stroke management. The way of working, learning or training within clinical placement for the individual physiotherapist might explain the existing variations between physiotherapists among their clinical practices in relation to the management of stroke. This is in line with the international classification of functioning, disability, and health (ICF) model which emphasizes that environmental factors along with the individuals' needs should be taken into account when delivering therapeutic interventions for people with stroke.

\section{End users}

Findings from this study indicated that the majority of the physiotherapists are aware of the relevance of the implementation. However, they emphasized the need to have the necessary skills to utilize available resources. Evidence from previous studies supported the claims of the current study that demonstrated how the positive attitude toward 
implementation was linked to other factors such as knowledge, level of education, skills, and knowledge.

Findings from the focus group reported that one method mostly employed with clinical practice was "experiential evidence". The findings in the current study match findings from several studies with respect to "expert opinion". 7,10,12 These studies reported that expert practice in physiotherapy had implications for physiotherapist practice, education, and implemention of evidence.

\section{Patient factors}

The practice of involving parents/family in the implementation of evidence into practice is usually seen as vital in clinical practice for stroke rehabilitation. Findings from the current study demonstrated difficulty in translating rehabilitation programs by stroke. This indicates a problem of inconsistencies in the therapeutic approach carried out in homes and hospitals. Similarly, evidence from a previous study reported that one of the major challenges facing patient treatment was the application of motor skills outside the therapeutic environment. ${ }^{18}$

Additionally, it was noted that integration of the research evidence into practice was influenced by the voicing of patients to accept intervention. ${ }^{19}$ The current study found patient factors to be one of the reasons for not using evidence on stroke rehabilitation.

\section{Transferability of evidence}

One of the central issues evident in the implementation of evidence in stroke rehabilitation was feasibility. Findings from this study demonstrated that the choice of appropriate evidence to match the local circumstance in the clinical practice was crucial in shaping the direction of the evidence in stroke rehabilitation. Previous studies reported the need for intervention activities to be implemented as intended using a similar popular population in an appropriate setting in a trial to ensure fidelity. ${ }^{32,34}$ Further previous research reported that one of the main reasons that physiotherapists failed to implement the guidelines into practice was due to lack of confidence in research findings and how they could be utilized an individual's work environment. ${ }^{13}$

\section{Conclusion}

The survey and focus group (mixed-methods) in this study were a useful way to provide a conceptual framework to promote the implementation of EBP in stroke rehabilitation. This method helped to overcome identified barriers and enhance enablers within the specific setting as outlined in the framework. Also, it helped to establish what influenced staff choices when classifying evidence and recommendations for stroke rehabilitation in the local context, the Kingdom of Saudi Arabia.

The findings derived from the key thematic areas provided comprehensive, rigorous, and systematic approaches to highlight any inconsistencies in the implementation of evidence-based stroke rehabilitation.

The suggested framework can provide the elements of successes needed for the implementation process of evidence-based stroke rehabilitation for the Kingdom of SaudiArabia's physiotherapists. Besides, the logical framework for the implementation process to fit a particular context will ascertain that the end product meets the stipulated needs. Including stroke patients and other stroke rehabilitation team members such as doctors, occupational therapists and nurses in future studies would expand our understanding in the implementation process of multidisciplinary team practice.

\section{Acknowledgments}

The author received no financial support for the research, authorship, and/or publication of this article.

\section{Disclosure}

The author reports no conflicts of interest in this work.

\section{References}

1. Rycroft-Malone J, Fotenia M, Bick D, Seers K. Protocol based care evaluation project report for the national co-ordinating centre for $N H S$ service delivery and organisation $R \& D$ (NCCSDO) SDO/78/2004. London: HMSO; 2007. doi:10.1094/PDIS-91-4-0467B

2. Scurlock-Evans L, Upton P, Upton D. Evidence-based practice in physiotherapy: a systematic review of barriers, enablers and interventions. Physiotherapy. 2014;100(3):208-219. doi:10.1016/j. physio.2014.03.001

3. Silva T, Costa L, Garcia A, Costa L. What do physical therapists think about evidence-based practice? A systematic review. Man Ther. 2014;20(3):388-401. doi:10.1016/j.math.2014.10.009

4. Scholten-Peeters G, Beekman-Evers M, van Boxel A, et al. Attitude, knowledge and behaviour towards evidence-based medicine of physical therapists, students, teachers and supervisors in the Netherlands: a survey. J Eval Clin Pract. 2011;19(4):598-606. doi:10.1111/j.13652753.2011.01811.X

5. Nilsagard Y, Lohse G. Evidence-based physiotherapy: a survey of knowledge, behaviour, attitudes and prerequisites. Adv Physiother. 2010;12(4):179-186. doi:10.3109/14038196.2010.503812

6. McEvoy M, Williams M, Olds T. Evidence based practice profiles: differences among allied health professions. BMC Med Educ. 2010;10 (1):69-76. doi:10.1186/1472-6920-10-69

7. Manns P, Darrah J. Linking research and clinical practice in physical therapy: strategies for integration. Physiotherapy. 2006;92(2):888-894. doi:10.1016/j.physio.2005.09.006 
8. Karin H, Staes F, Goedhuys J, Aertgeerts B. Obstacles to the implementation of evidence-based physiotherapy in practice: a focus group-based study in Belgium (Flanders). Physiother Theory Pract. 2009;25(7):476-488. doi:10.1080/09593980802661949

9. Iles R, Davidson M. Evidence based practice: a survey of physiotherapists' current practice. Physiother Res Int. 2006;11(2):93-103.

10. Schreiber J, Stern P. A review of the literature on evidence -based practice in physical therapy. Internet $J$ Allied Health Sci Pract. 2005;3(4):1540-1580.

11. Hamzat T, Amusat N. Belief and participation with clinical physiotherapists in research. S Afr J Physiother. 2002;58(2):32-34. doi:10.4102/sajp. v58i2.121

12. Barnard S, Wiles R. Evidence-based physiotherapy. Physiotherapists' attitudes and experiences in the Wessex area. Physiotherapy. 2001;78 (3):115-124. doi:10.1016/S0031-9406(05)61078-4

13. Upton D. Clinical effectiveness and EBP 2: attitudes of health-care professionals. $\mathrm{Br} J$ Ther Rehabil. 1999;6(1):26-30. doi:10.12968/ bjtr.1999.6.1.14019

14. Turner P, Whitfield T. Physiotherapists' reasons for selection of treatment techniques: a cross-national survey. Physiother Theory Pract. 1999;15(4):235-246. doi:10.1080/095939899307649

15. Salbach N, Jaglal S, Korner-Bitensky N, Rappolt S, Davis D. Practitioner and organizational barriers to evidence-based practice of physical therapists for people with stroke. Phys Ther. 2007;87 (10):1284-1303. doi:10.2522/ptj.20070040

16. Mclnerney P, Suleman F. Exploring knowledge, attitudes, and barriers toward the use of evidence-based practice amongst academic health care practitioners in their teaching in a South African university. A Pilot Study. Worldviews Evid Based Nurs. 2010;7(2):90-97.

17. Dannapfel P, Peolsson A, Nilsen P. What supports physiotherapists' use of research in clinical practice? A qualitative study in Sweden. Implement Sci. 2013;8(1):31-52. doi:10.1186/1748-5908-8-31

18. Loupis Y, Faux S. Family conferences in stroke rehabilitation: a literature review. J Stroke Cerebrovas Dis. 2013;22(6):883-893 doi:10.1016/j.jstrokecerebrovasdis.2012.12.003

19. Bower K, Gustafsson L, Hoffmann T, Barker R. Self-management of upper limb recovery after stroke: how effectively do occupational therapists and physiotherapists train clients and carers? Br J Occup Ther. 2012;75(4):180-187. doi:10.4276/030802212X13336366278130

20. Pollock A, Legg L, Langhorne P, Sellars C. Barriers to achieving evidence-based stroke rehabilitation. Clin Rehabil. 2000;14 (6):611-617. doi:10.1191/0269215500cr369oa

21. Scott S, Albrecht L, O'Leary K, et al. Systematic review of knowledge translation strategies in the allied health professions. Implement Sci. 2012;7(1):70-87. doi:10.1186/1748-5908-7-70

22. Eccles M, Grimshaw J, Walker A, Johnston M, Pitts N. Changing the behavior of healthcare professionals: the use of theory in promoting the uptake of research findings. $J$ Clin Epidemiol. 2005;58 (2):107-112. doi:10.1016/j.jclinepi.2004.09.002

23. Rycroft-Malone J, Bucknall T. Models and Frameworks for Implementing Evidence-Based Practice. 2nd ed. UK: Wiley Blackwell; 2010.
24. Tabak R, Khoong E, Chambers D, Brownson R. Bridging research and practice: models for dissemination and implementation research. Am J Prev Med. 2012;43(3):337-350. doi:10.1016/j. amepre.2012.05.024

25. Stetler C. Refinement of the Stetler/Marram model for application of research findings to practice. Nurs Outlook. 1994;42(1):15-25.

26. Logan J, Graham I. Toward a comprehensive interdisciplinary model of health care research use. Sci Community. 1998;20(2):227-246. doi:10.1177/1075547098020002004

27. Kitson A, Rycroft-Malone J, Harvey G, McCormack G, Seers K, Titchen A. Evaluating the successful implementation of evidence into practice using PARIHS framework: theoretical and practical challenges. Implement Sci. 2008;3(1):1-12. doi:10.1186/1748-5908-3-1

28. Rycroft-Malone J, Kitson A, Harvey G, et al. Ingredient for change: revisiting a conceptual framework. Qual Saf Health Care. 2002;11 (2):174-180. doi:10.1136/qhc.11.2.174

29. Damschroder L, Aron D, Keith R, Kirsh S, Alexander J, Lowery J. Fostering implementation of health services research findings into practice: a consolidated framework for advancing implementation science. Implement Sci. 2009;4(1):50-65. doi:10.1186/1748-5908-4-50

30. Graham I, Logan J, Harrison M, et al. Lost in knowledge translation: time for a map? J Contin Educ Health Prof. 2006;26(1):13-24. doi:10.1002/chp.47

31. Bekkering G, Hendriks H, Van Tulder M, et al. Effect on the process of care of an active strategy to implement clinical guidelines on physiotherapy for low back pain: a cluster randomised controlled trial. Qual Saf Health Care. 2005;14(2):107-112. doi:10.1136/ qshc. 2003.009357

32. Van der Wees P, Moore A, Powers C, Stewart A, Nijhuis-van der Sanden M, De Bie R. Development of clinical guidelines in physical therapy: perspective for international collaboration. Phys Ther. 2011;91(10):1551-1563. doi:10.2522/ptj.20100305

33. Hanchard N, Goodchild L, Thompson J, O'Brien T, Davison D, Richardson C. Evidence-based clinical guidelines for the diagnosis, assessment and physiotherapy management of contracted (frozen) shoulder: quick reference summary. Physiotherapy. 2012;98 (2):117-120. doi:10.1016/j.physio.2012.01.001

34. Van der Wees P, Jamtvedt G, Rebbeck T, de Bie R, Dekker J, Hendriks E. Multifaceted strategies may increase implementation of physiotherapy clinical guidelines: a systematic review. Aus J Physiother. 2008;54(4):233-241.

35. Menon A, Korner-Bitenskey N, Kastner M, McKibbon K, Straus S. Strategies for rehabilitation professionals to move evidence-based knowledge into practice: a systematic review. $J$ Rehabil Med. 2009;41(13):1024-1032. doi:10.2340/16501977-0451

36. Jans M, Schellevis F, Le Coq E, Bezemer P. Health outcomes of asthma and COPD patients: the evaluation of a project to implement guidelines in general practice. Int J Qual Health Care. 2001;13 (1):17-25. doi:10.1093/intqhe/13.1.17
The Journal of Multidisciplinary Healthcare is an international, peerreviewed open-access journal that aims to represent and publish research in healthcare areas delivered by practitioners of different disciplines. This includes studies and reviews conducted by multidisciplinary teams as well as research which evaluates the results or conduct of such teams or healthcare processes in general. The journal covers a very wide range of areas and welcomes submissions from practitioners at all levels, from all over the world. The manuscript management system is completely online and includes a very quick and fair peer-review system. Visit http://www.dovepress.com/testimonials. php to read real quotes from published authors. 